\title{
Sistematización del Proyecto CARS ejecutado por la URACCAN, Municipio de Puerto Cabezas
}

\section{CARS Project Systematization executed by URACCAN, Puerto Cabezas Municipality}

\author{
Sesiah Lizani Davis Gram \\ Yilda Vanessa López Gutiérrez ${ }^{2}$ \\ Fanny Carina Flores Omier ${ }^{3}$ \\ Keith Sankara Narvaez Ismael ${ }^{4}$ \\ Elizabeth Salomón McCLean ${ }^{5}$ \\ Miguel Núñez ${ }^{6}$
}

\section{Resumen}

El proyecto Acción Comunitaria para la Lectura y Seguridad (CARS por sus siglas en inglés), tuvo el propósito de mejorar los resultados de la lectura y contribuir a la reducción de la inseguridad ciudadana de la niñez en preescolar y en los primeros grados de educación primaria. En este sentido se presenta la sistematización de la experiencia y los resultados del proyecto en los grupos meta. Es un estudio de carácter cualitativo, con enfoque participativo de los actores involucrados de esta experiencia en la implementación de las metodologías: Aprendo-Práctico-Aplico (APA) y Fonético-Analítico-Sintético (FAS); así mismo, las acciones desarrolladas para la identificación y abordaje de factores de inseguridad en la escuela y la comunidad, trata de personas y violencia basada en género, en once escuelas del municipio de Puerto Cabezas en el 2018. Los resultados de la sistematización evidencian los procesos desarrollados, logros alcanzados y las lecciones aprendidas en la aplicación de las metodologías pedagógicas que servirán para futuras experiencias.

Palabras clave: educación; fluidez; comprensión lectora; seguridad ciudadana.

\section{Abstract}

The Community Action for Reading and Safety (CARS) Project aimed to improve reading results and contribute to reducing citizen insecurity of children in preschool and in the first grades of primary education. In this sense, the systematization of the experience and the results of the project in the target groups is presented. It is a qualitative study, with a participatory approach of the actors involved in this experience, focusing on the implementation of the methodologies: I Do, You Do, We Do Method (APA for the Spanish Acronym) and Phonetic-Analytical-Synthetic (FAS for its acronym in Spanish); likewise, the actions developed to identify and address insecurity factors in the school and the community, human trafficking and gender-based violence in eleven schools in Puerto Cabezas municipality in 2018. The results of the systematization show the developed processes, achieved goals and the lessons learned in the application of the pedagogical methodologies that will serve for future experiences.

\footnotetext{
1 Máster en Planificación y Desarrollo Regional con Identidad, Coordinadora Observatorio de la autonomía de la URACCAN Recinto universitario Bilwi. Correo: sesidavis85@yahoo.es ORCID: https://orcid.org/0000-0003-3718-1901

2 Licenciada en Sociología con mención en Autonomía. URACCAN. Correo vanessalg1796@gmail.com; ORCID: https://orcid.org/0000-0002-3856-4560

3 Licenciada en Sociología con mención en Autonomía. URACCAN. Correo hannyaomier@gmail.com

4 Máster en Planificación y Desarrollo Regional con Identidad, Coordinador Cooperación Externa de la Universidad de las Regiones Autónomas de la Costa Caribe Nicaragüense - Recinto Universitario Bilwi. Correo: cooperacion.bilwi@uraccan.edu.ni; ORCID: https://orcid.org/0000-0003-3268-2643

5 Máster en Planificación y Desarrollo Regional con Identidad. Coordinadora Instituto de Promoción e Investigación Lingüística y Revitalización Cultural de la URACCAN Recinto Universitario Bilwi. Correo: elisalomon12@yahoo.es ORCID: https://orcid.org/0000-0001-8650-8857

6 Licenciado en Economia, Subdirector DevTech Systems, Inc. Correo: mnunez@devtechsys.com
} 
Keywords: Education; fluency; reading comprehension; citizen security.

\section{Introducción}

En el 2018, la URACCAN ejecuta el proyecto Acción Comunitaria para la Lectura y Seguridad" (CARS), el proyecto se enmarca en el ámbito educativo de trabajo con escuelas de pre-escolar y primaria enfocando principalmente el área de lengua y literatura. Fue ejecutado en el 2018 en el marco del convenio No. AID 524-C-13-0001 entre DevTech Systems, Inc. y la URACCAN, financiado por la Agencia de los Estados Unidos para el Desarrollo Internacional (USAID). Se basó en la metodología APA y FAS con el ánimo de contribuir a mejorar los resultados de lectura temprana y la reducción de la inseguridad ciudadana en 11 escuelas del Municipio de Puerto Cabezas.

Sistematizar esta experiencia es de suma importancia ya que permite visualizar y compartir los procesos desarrollados en el marco del proyecto, el análisis de los logros alcanzados y los aprendizajes que se generaron. A nivel institucional, es vista como un aporte a la accesibilidad y mejora de la educación regional sobre todo en zonas rurales alejadas. La sistematización se fundamenta en la metodología participativa. Se realizaron grupos focales con maestros y facilitadores del proceso, diálogo con infantes y entrevistas con los padres y madres de familia, directores (as) de los centros educativos y algunas autoridades del ministerio de educación.

El aporte de la sistematización demuestra que, la aplicación de las metodologías pedagógicas en los primeros grados de primaria y a través del seguimiento constante en las escuelas se logró un avance significativo en las habilidades de fluidez y comprensión lectora de primero a tercer grado, esto se documentó con la aplicación de Pruebas Periódicas de Lectura (PPL) quedando fortalecidas las capacidades de docentes en su aplicación ya que regularmente fueron aplicadas por ellos mismos. Otro de los logros alcanzados fue la retención escolar en un $94 \%$ en todos los grados y el involucramiento de padres y madres de familia.

\section{Revisión de literatura}

\section{La metodología APA}

Según el (MINED, 2009) la metodología Aprendo Practico y Aplico que es utilizada en el ámbito de la educación primaria en el país, es visualizada como una tendencia moderna que se fundamenta en el enfoque constructivista de la teoría piagetiana, tiene como fin ofrecer una manera distinta de comprender como se construye el conocimiento en interacción con el medio, con la vida, en la interacción maestro-alumno-entorno, poniendo a prueba, saberes y experiencias previas, valores y actitudes (pág. 37).

\section{La metodología Fonético-Analítico y Sintético (FAS)}

Es un método mixto en el que combina simultáneamente lo sintético con lo analítico. Tiene tres componentes: el fónico, porque su base o fundamento está en el estudio del sonido, del habla viva; el analítico porque en el aprendizaje los niños tienen que dividir las oraciones en palabras, las palabras en silabas y las sílabas en sonidos; y el sintético porque durante su desarrollo los niños aprenden a integrar de nuevo las partes hasta llegar a recomponer el todo (Lopez y Mora, 2016, pág. 37).

\section{Etapas del desarrollo cognitivo}

La teoría de Jean Piaget es fundamental en la psicopedagogía constructivista (del aprendizaje cognitivo y social) para comprender las etapas del desarrollo cognitivo, según esta teoría el ser humano adquiere el conocimiento a partir de su interacción con el medio y difiere según procesos, etapas o las cuales denominó como "estadios" del desarrollo. Saldarriaga, Bravo, y Loor (2016) citando a Piaget (1969) indican que el desarrollo cognitivo o cognoscitivo es un proceso continuo de construcción y de conocimiento que inicia 
desde la niñez y se manifiesta en una serie de etapas que pueden variar en distintos grupos poblacionales de acuerdo al contexto en que se desarrolle su formación y la cultura que tengan.

De acuerdo a la teoría piagetiana, el desarrollo cognitivo ocurre en cuatro etapas según ciertos niveles de edad y siguen una secuencia invariable. Según el grupo meta del proyecto, nos centraremos en la segunda (de 2 a 7 años) y tercera etapa (de 7 a 11 años). Plantea que en la segunda etapa los niños -comprende la edad de pre-escolar ( 5 y 6 años) - es más intuitivo, puede usar símbolos y palabras para pensar y busca una solución intuitiva de los problemas. En la tercera etapa -edades en primeros grados de primaria- los niños son más prácticos, de pensamiento lógico ligado a fenómenos y objetos del mundo real. En efecto, las metodologías APA y FAS buscan potenciar estas habilidades en edades tempranas.

\section{Métodos y materiales}

El estudio es de carácter cualitativo, tuvo un enfoque participativo con los actores involucrados. Se realizaron entrevistas individuales, grupos focales y diálogo con los niños de educación primaria y pre-escolar. El estudio abarcó las 11 escuelas atendidas por la URACCAN en el Municipio de Puerto Cabezas: Emanuel Mongalo y Rubio (comunidad de Sahsa), Pedro Bello (comunidad Mani Watla), Raúl Martínez (comunidad Sangnilaya), Andrés Castro (comunidad Panua), Rafaela Herrera (comunidad Iltara), Escuela Trimbach (Comunidad Krukira), Llano Verde (comunidad Tuapi), Nueva Jerusalén 1, Nueva Jerusalén 2, Julio Bucardo y Doris Robb Gabay, éstas últimas cuatro ubicadas en el área urbana de la ciudad de Bilwi.

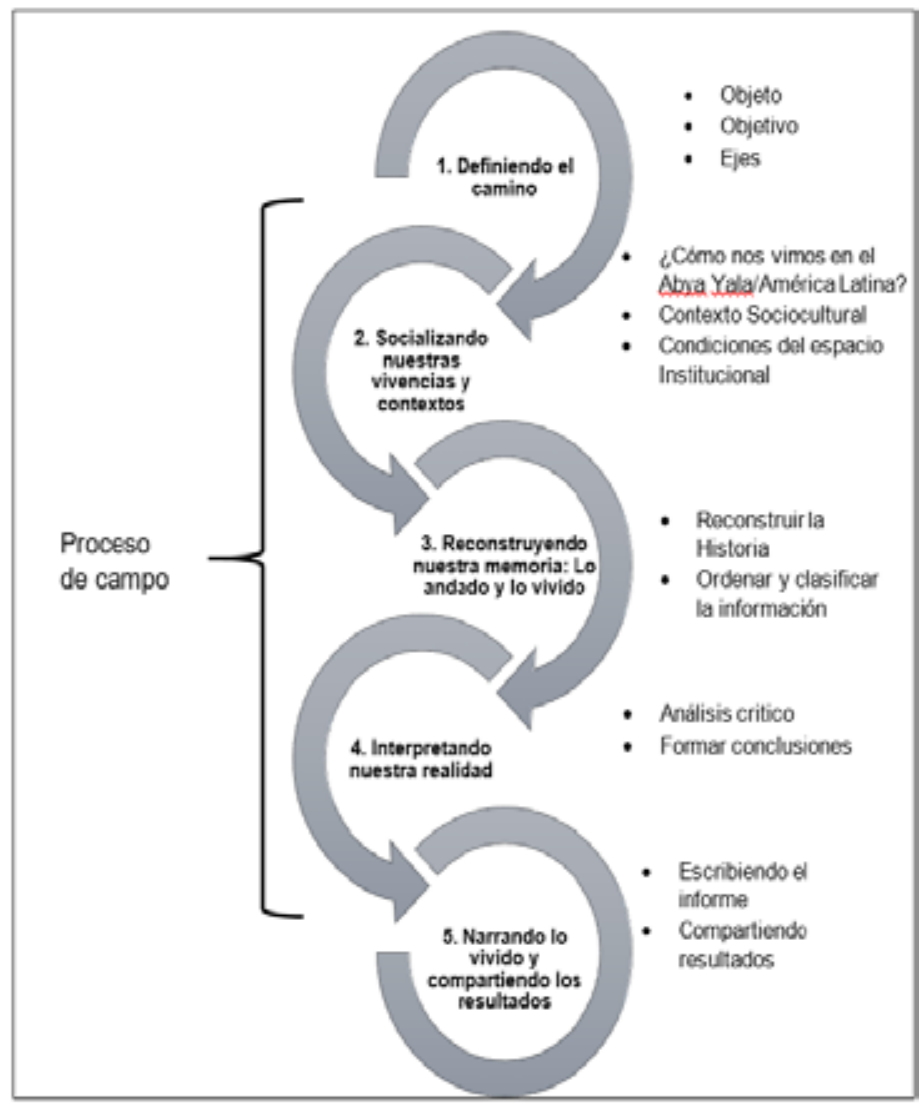

Gráfico 1. Pasos metodológicos para la recreación, URACCAN 2017.

El trabajo de campo para reconstrucción de la experiencia y la documentación de percepciones vinculadas a cada una de las fases del proyecto se realizó con 142 protagonistas (entre maestros/as de todos los niveles educativos, directores, padres y madres de familia y algunos niños 93 del sexo femenino y 49 del 
sexo masculino. Según la identidad étnica, 114 son de la etnia mískitu, 27 son mestizos y 1 es de la etnia mayangna.

El proceso de la sistematización se realizó en cinco pasos metodológicos utilizando la metodología de la (URACCAN, 2017): "Sendas para recrear y caminar la experiencia con perspectiva intercultural de género" (Gráfico 1).

\section{Resultados de la sistematización}

El proyecto CARS se basó en procesos de acompañamiento y monitoreo constante por el personal técnico de la URACCAN a los docentes de educación primaria (preescolar, primero, segundo y tercer grado) para garantizar la implementación de la metodología APA en los tres primeros grados de primaria y la metodología FAS que contribuyó a desarrollar habilidades de lectoescritura inicial en niños del tercer nivel de pre-escolar. El abordaje metodológico del proyecto inició con la selección propuesta de las escuelas beneficiarias y la coordinación con las autoridades educativas y comunitarias. Luego de eso, el desarrollo de las acciones según los dos componentes del proyecto: i) "Lectura formal y no formal" ii) "Participación comunitaria" .

\section{Selección de los centros educativos}

La selección de los centros se hizo en coordinación con el Ministerio de Educación (MINED). Primero, fue una pre-selección de 15 escuelas de primaria y pre-escolar de los Municipios de Puerto Cabezas y Waspam. Intentando priorizar escuelas bilingües y zonas de difícil acceso en el área rural para el Ministerio de Educación por la falta de recursos o de personal técnico. "En las reuniones con la URACCAN, una de nuestras prioridades para la selección de las escuelas fue potenciar el trabajo donde el MINED no tenía la capacidad de intervención, por razones financieras o de recursos humanos" (entrevista Tessia Torrez, Delegada Departamental del MINED en Bilwi).

Luego, sobre la base de las escuelas pre-seleccionadas Devtech y la URACCAN, se seleccionaron 10 escuelas ( 6 del área rural en Puerto Cabezas y 4 en Waspám) tomando en cuenta la factibilidad de una ruta para el acompañamiento y asistencia técnica en las escuelas. Sin embargo, en el 2018 debido a un reajuste financiero se decidió la intervención de la URACCAN únicamente en el Municipio de Puerto Cabezas en 11 escuelas: 7 en el área rural y 4 en el área urbana. Las escuelas atendidas son:

Tabla 1. Escuelas atendidas por el proyecto CARS

\begin{tabular}{|l|l|l|l|}
\hline \multicolumn{2}{|c|}{ Área Rural } & \multicolumn{2}{c|}{ Área Urbano } \\
\hline \multicolumn{1}{|c|}{ Comunidad } & \multicolumn{1}{c|}{ Escuela } & \multicolumn{1}{c|}{ Bilwi } & \multicolumn{1}{c|}{ Escula } \\
\hline Sahsa & Emanuel Mongalo y Rubio & Bilwi & Nueva Jerusalén 1 \\
\hline Mani Watla & Pedro Bello & Bilwi & Nueva Jerusalén 2 \\
\hline Sangnilaya & Raúl Martínez & Bilwi & Julio Bucardo \\
\hline Panua & Andrés Castro & Bilwi & Doris Robb Gabay \\
\hline Iltara & Rafaela Herrera & & \\
\hline Krukira & Trimbach & & \\
\hline Tuapi & Llano Verde & & \\
\hline
\end{tabular}

\subsection{Coordinación y articulación con las autoridades}

En tanto proyecto educativo y con el ánimo de trabajar conjuntamente con los entes rectores de la educación en el municipio, fue clave en todo el proceso la coordinación y articulación, primero con la Dirección del Sistema Educativo Autonómico Regional (SEAR), la Delegación Departamental y Municipal 
del Ministerio de Educación (MINED), quienes avalaron el proyecto en las comunidades seleccionadas para mejorar los resultados de la lectura en la educación primaria.

Así mismo, de acuerdo a la organización comunitaria y tradicional en las comunidades mískitu también se realizó la coordinación con las autoridades territoriales y comunitarias, los directores (as) y docentes de los centros educativos para presentar las acciones que se iban a estar trabajando en las escuelas.

\section{Desarrollo de los componentes del proyecto}

El proyecto como tal trabajó en dos componentes: primero, el componente de "Lectura formal y no formal" comprendió el acompañamiento y monitoreo a los docentes dentro del aula de clase para garantizar la aplicación de las metodologías APA y FAS. El segundo componente de "Participación comunitaria" trabajó el enfoque de la seguridad ciudadana con padres y madres de familia y actores comunitarias para reducir los factores de inseguridad en la escuela y en la comunidad.

\section{Componente 1. Lectura formal y no formal}

El desarrollo del componente de "Lectura formal y no formal" tuvo como punto de partida la capacitación al equipo técnico para la apropiación de las metodologías e instrumentos pedagógicos. Luego de eso, organizar y facilitar las condiciones y materiales necesarios para la atención integral de niños fue importante porque a la par de las metodologías y estrategias brindadas, los docentes disponían de los recursos para mejorar las condiciones del ambiente escolar. El fortalecimiento de capacidades a docentes incluyó el asesoramiento para la elaboración de rincones de lectura, observaciones a pie de aula con el instrumento de observación "stalling" y talleres de réplica para la aplicación de Pruebas Periódicas de Lectura (PPL) y sobre la metodología APA, FAS y guías metodológicas.

\section{a. Capacitación al equipo técnico y a docentes}

El fortalecimiento de capacidades al equipo técnico y a docentes fue clave para asegurar el éxito de las metodologías y el seguimiento adecuado en las escuelas. Se desarrollaron en dos momentos: La primera capacitación fue en el 2017 en el uso de los componentes de lectura y las metodologías, participaron el equipo técnico y docentes de los tres primeros grados de primaria. Fue asesorado por una especialista en lecto-escritura y educación multicultural. En segundo momento, Devtech capacitó al equipo técnico de la URACCAN en el uso y manejo de las estrategias metodológicas, aplicación de Pruebas Periódicas de Lectura y uso del instrumento de observación como parte del fortalecimiento de capacidades para el acompañamiento y asistencia técnica en las escuelas.

Una de las valoraciones que hacen los docentes es que, si bien son metodologías que promueve el MINED, la capacitación facilitó formas prácticas sobre cómo implementarlas en el aula de clase. "Para mí, ha sido como un reforzamiento porque tiene relación con lo que mandata el MINED y con el proyecto he aprendido nuevas estrategias de enseñanza para utilizar en el aula de clases" (docente de la escuela Julio Bucardo, Bilwi).

\section{b. Inscripción y registro de matrícula}

En el marco del proyecto, la inscripción de los protagonistas en base a la matrícula oficial es fundamental para tener un registro del número de beneficiaros atendidos por el proyecto en cada una de las escuelas y sobre la base de este registro valorar los resultados y evolución de los indicadores de fluidez y comprensión lectora durante todo el proceso. De las 11 escuelas fueron beneficiados un total de 1,885 protagonistas, 520 son estudiantes de tercer grado, 525 de segundo grado, 592 de primer grado y 218 del tercer nivel de preescolar. De este total, el 62\% de los protagonistas son del sexo femenino y $38 \%$ son del sexo masculino, la mayoría $(1,590)$ son de la etnia mískitu, 225 mestizos, 39 creoles y 1 mayangna. 


\section{c. Dotación de materiales didácticos y pedagógicos}

Para apoyar la implementación de las metodologías, un factor importante fue la entrega de materiales didácticos a docentes y a estudiantes de las 11 escuelas ya que posibilitó herramienta a los docentes y a las escuelas que muchas veces no tienen esa accesibilidad. Bajo esta perspectiva, a los docentes de primaria se facilitó guías de estrategias educativas tituladas "Leyendo para la Vida" de acuerdo a cada nivel educativo (primero, segundo y tercer grado) para apoyar el proceso de enseñanza-aprendizaje dentro del aula de clase y materiales didácticos tales como cartulinas, papeles, pegamento, colores, entre otros los cuales sirvieron para habilitar pequeñas bibliotecas en las escuelas para que sean espacios de aprendizaje y práctica de lectura.

A docentes del III nivel de pre-escolar, se entregó una serie de guías para el desarrollo de capacidades de los niños titulados: i) Somos felices que se enfoca en el área socio-afectiva ii) Somos sociales iii) Somos capaces orientado a fortalecer el área cognitiva o del conocimiento iv) Somos movimiento y avanzamos enfocado en el área psicomotora. Así también se entregaron guías de trabajo con padres y madres de familia. Para fomentar el hábito de la lectura, a los niños de primaria se entregaron textos de Lengua y Literatura de la colección "Nachos de Nicaragua" y libros de cuento según el nivel educativo. Aunque la mayoría de los textos y cuentos eran en español, algunos de los cuentos estaban en la lengua mískitu.

\section{d. Acompañamiento y asesorías técnicas a docentes}

El acompañamiento y asesorías técnicas del personal técnico a los docentes en cada una de las escuelas es un proceso clave en la implementación de las metodologías, los componentes de la lectura (fluidez lectora, comprensión lectora, código alfabético, conciencia fonológica) y el uso de estrategias metodológicas para la enseñanza de la lectura en las clases de: lengua y literatura; y Lengua Indígena o Lengua 1 (en el caso de las escuelas bilingües). Comprendió observaciones a pie de aula, asesorías técnicas individuales y grupales a docentes; el acompañamiento para la aplicación de Pruebas Periódicas de Lectura (PPL) que fortalecieron el proceso de enseñanza-aprendizaje en el área de Lengua y Literatura para el desempeño del alumno/a.

En lo manifestado por los docentes, el acompañamiento es visto como un reforzamiento al trabajo docente. La aplicación de PPL, se valora como nuevo conocimiento para evaluar de forma práctica y rápida el aprendizaje la lectura, la evolución de la fluidez y comprensión lectora en los estudiantes. "Me ha ayudado a mejorar la forma de dar la clase, organizar el plan de clase y utilizar estrategias creativas que motiven a los estudiantes a participar manteniendo la dinámica en las aulas de clases" (docente de primer grado, escuela Emmanuel Mongalo en Sahsa).

\section{e. Observaciones a pie de aula}

Es uno de los principales instrumentos que se utilizó para el seguimiento diferenciado en los grados de primaria y del tercer nivel de pre-escolar, pero focalizadas a las asignaturas de Lengua y Literatura y Lengua Indígena o Lengua 1 durante un periodo de 90 o 45 minutos, lo cual permitió ir observando las debilidades en el proceso de enseñanza-aprendizaje que dieron pautas para la retroalimentación en conjunto con el/la docente para enriquecer el proceso educativo.

En los grados de primaria se utilizó el instrumento Stalling para observar la metodología que utiliza el docente para el desarrollo de la lectura, la ambientación del aula, organización del tiempo, el componente desarrollado, ciertas condiciones de ventilación, limpieza y el aprendizaje del estudiante en base a la metodología empleada por el docente, así como el porcentaje de tiempo del docente en las actividades académicas, no académicas y de involucramiento. En pre-escolar se utilizó un instrumento menos estandarizado que el Stalling, observando la ambientación de aula y actividades que promueve el docente en el aula de clases para desarrollar el área afectiva, cognitiva y psicomotora. 


\section{f. Asesorías técnicas a docentes}

En el caso de los docentes de pre-escolar, el equipo técnico desarrolló réplicas de capacitaciones y asistencia técnica en el uso y manejo de las guías de aprendizaje para desarrollar el área afectiva, cognitiva y psicomotora; así mismo, el uso de la guía metodológica para el trabajo con padres y madres de familia. Con los docentes de primaria, el equipo técnico apoyó en la elaboración de materiales didácticos, rincones de aprendizaje, pequeñas capacitaciones sobre el uso y manejo de los componedores colectivos e individuales, elaboración de planes creativos y participativos con actividades lúdicas y talleres de réplica para la aplicación de PPL.

\section{g. Aplicación de PPL}

La prueba periódica de lectura es un instrumento que evalúa la comprensión y fluidez lectora según el nivel educativo del estudiante. Estas evaluaciones se realizaron por trimestre en los tres grados de primaria por los docentes a una muestra de 10 estudiantes por grado. Durante el primer trimestre, las pruebas se realizaron en la lengua mískitu en las escuelas bilingües. Luego, en los siguientes trimestres, se aplicaron en español por la dificultad que presentaban los niños en leer en su lengua materna, lo cual se puede asociar a que normalmente las clases se desarrollan en español a pesar de que algunas sean escuelas bilingües se observa que la lengua materna se utiliza más de forma explicativa o para complementar, por una parte, por la escasez de materiales pedagógicos en el idioma materno, lo cual evidencia la necesidad de fortalecer las capacidades actualizaciones didácticas al personal docente para la enseñanza de la lengua materna.

\section{Componente 2. Participación comunitaria}

El segundo componente consistió en la promoción de eventos educativos de lectura, en cada una de las escuelas involucrando además de la comunidad educativa a los padres y madres de familia. Así también, se trabajó en procesos de reflexión sobre inseguridad ciudadana con padres y madres de familia y actores comunitarios para la reducción de los factores de riesgo que generan inseguridad en la escuela y en la comunidad.

\section{a. Promoción de la lectura}

Muchas veces en las escuelas, la educación se confina al trabajo dentro de las aulas de clase y poco se promueven actividades que motiven y donde los niños puedan mostrar su talento, además que poco se involucran los padres y madres de familia en los procesos educativos de sus hijos e hijas. Tratando de mejorar esta situación, el proyecto promovió distintas iniciativas como concursos de lectura y cuentos, competencias de murales, rincones de aprendizaje y la hora del cuento, todas estas estrategias para promover la lectura y escritura de los niños.

En algunas fechas efemérides (Día del Libro, Día del niño y Día de la Alfabetización) se realizaron competencias de murales, concursos de lectura donde los niños de primaria participaban en competencias de lecturas en voz alta, trabalenguas, adivinanzas y poesía. En el caso de pre-scolar participó con cantos, cuentos y dibujos logrando desarrollar importantes habilidades de expresión oral en ambos idiomas. En la valoración que hacen los docentes, estas actividades estimulan la creatividad y utilización de los medios naturales, se logró brindar herramientas sobre el uso de los medios naturales para promover la lectura. "Ahora veo que los niños ahora son más participativos, les motiva, lo ven como una competencia de ir mejorando cada día para participar y ganar premios. También elaboran poemas, cantos y cuentos con mucha creatividad" (profesora escuela Julio Bucardo en Bilwi).

\section{b. Escuelas de padres y madres}

La URACCAN consciente de la importancia del involucramiento de padres y madres de familia en los procesos educativos enfocando los problemas de inseguridad, trabajó la iniciativa de "Escuelas de padres 
y madres de familia" con el propósito de generar conciencia sobre importantes temas educativos que permitan el involucramiento de padres, madres y tutores afectivos en el proceso de enseñanza-aprendizaje. De igual forma se realizaron reflexiones sobre la identificación y el abordaje de la Trata de Personas y la Violencia Basada en Género, entre otros factores de riesgo para los niños (URACCAN, o1 Febrero al 31 de Diciembre, 2018).

\section{c. Documentación de historias de éxito}

En el marco del proyecto, los beneficiarios demostraron muchos cambios, ya sean niños, niñas, docentes, padres, madres de familia en su proceso de aprendizaje y de su vida personal. La URACCAN documentó 4 historias de éxito: una madre que a través de las EPM reconoce la importante de ayudar a su hija en las tareas de la escuela; dos docentes que aprovechan los materiales didácticos y estrategias metodológicas para dinamizar las clases, así mismo, la historia de una niña de 7 años de edad que narra el antes y después en la práctica de la lectura con su libro de cuentos. Esto permitió visualizar los resultados positivos o cambios generados en la vida de los protagonistas, un proceso clave de divulgación.

\section{d. Comisiones de análisis}

Las comisiones de análisis son estructuras integradas por padres y madres de familia en cada una de las escuelas beneficiarias dedicadas a identificar y buscar solución a factores generadores de riesgo en la escuela y la comunidad. Como miembro de la comisión de análisis: "Nosotros visitamos la escuela y apoyamos el trabajo de los docentes, ayudamos a controlar la disciplina pues es difícil para una sola persona controlar casi 30 estudiantes, también velamos por el aprendizaje de los niños y el rendimiento académico" (madre de familia de la escuela Emmanuel Mongalo, Sahsa).

\section{Logros alcanzados en la fluidez y comprensión lectora}

Para observar la evolución de los resultados de fluidez y comprensión lectora durante el 2018, se aplicaron Pruebas Periódicas de Lectura (PPL) a estudiantes del primero, segundo y tercer grado, en tres momentos: Durante el primer trimestre (mes de junio), el segundo trimestre (mes de septiembre) y el tercer trimestre (mes de octubre). En total se aplicó 1, 442 PPL (todas en español), 167 varones y 161 mujeres en las pruebas iniciales, 238 varones y 262 mujeres en las pruebas intermedias; 288 varones y 281 mujeres en las pruebas finales. En cada uno de los momentos se selección una muestra de 10 estudiantes por grado.

\section{a. Fluidez lectora}

En todos los grados académicos, se observa un incremento de palabras correctas leídas por minuto (de aquí en adelante PCPM) entre la medición inicial y final. Los niños de primer grado que apenas está comenzando a leer, pasan de 13 PCPM en la medición intermedia a 29.5 PCPM en la medición final. Segundo grado, pasa de 10.99 PCPM en la prueba inicial a 29.5 palabras correctas leídas por minuto en la prueba final. Tercer grado, pasa de 30.95 PCPM a 58 PCPM en la prueba final.

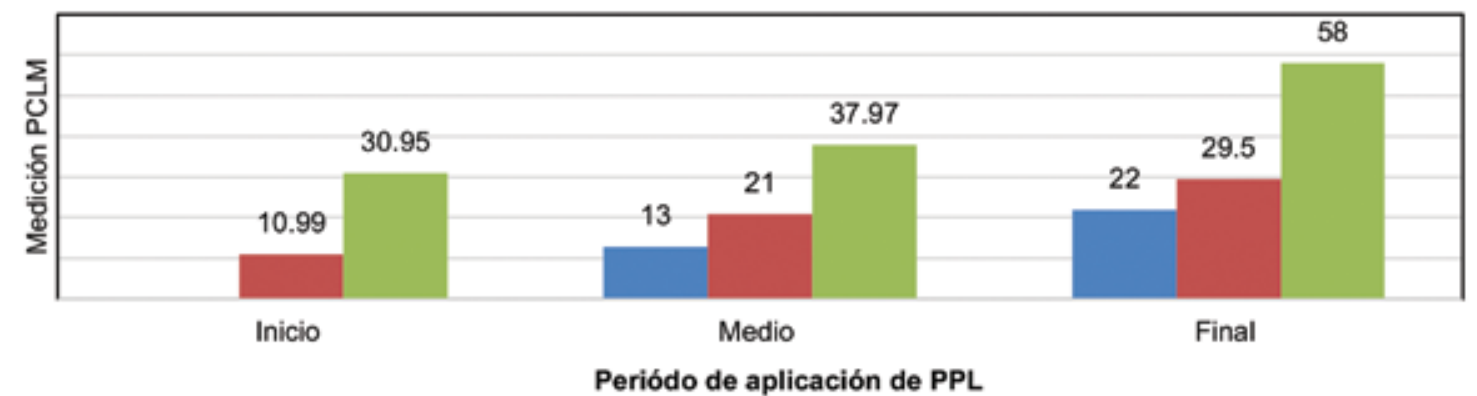

\pm Primero $=$ Segundo $=$ Tercero

Gráfico 2. Resultado de Fluidez lectora por grado y período.

Fuente: Monitoreo y Evaluación del proyecto CARS en la URACCAN. Reporte noviembre 2018. 
De acuerdo a los estándares o metas internacionales consensuados para los países en vías de desarrollo indican que "para finales de primero y segundo grado el niño/a debe leer aproximadamente 6o palabras correctas por minuto" (USAID, 2009, pág. 65). Bajo esta premisa, los resultados alcanzados si bien muestran un crecimiento gradual y significativo, ninguno de los grados académicos alcanza el promedio de 60 PCPM en la evaluación final porque tiene que ver con un tema estructural de la educación de los grados en que el niño/a inicia la escuela y que se necesita potenciar estas prácticas metodológicas y de evaluación en todos los centros educativos para elevar la calidad de la educación.

En relación a los datos por sexo, tanto varones como mujeres incrementaron la mediana de palabras leídas por minuto. Del total de pruebas aplicadas, los varones pasaron de 17 PCPM en la prueba inicial a 25 PCPM en la prueba final. Las mujeres, pasaron de 19.95 PCPM en la medición inicial a 37 PCPM en la medición final. Según la identidad étnica, el grupo mestizo presenta mejores resultados, lo cual puede asociarse a que el español es su lengua materna. No tanto así en las escuelas de las zonas rurales indígenas donde predomina la población indígena mískitu, pero, son pocos en comparación con las matrículas de las escuelas urbanas.

\section{b. Comprensión lectora}

En la comprensión lectora, se evidencian avances significativos en todos los grados académicos. Por ejemplo, en la prueba intermedia aplicada a primer grado, los estudiantes respondieron correctamente el $33.33 \%$ de las preguntas, incrementando a un 50\% en la medición final. Destaca segundo grado, que pasa de $66.67 \%$ preguntas contestadas correctamente en la prueba intermedia a un $100 \%$ en la prueba final. Tercer grado, pasa de un 50\% en la prueba inicial a un $80 \%$ en la prueba final.

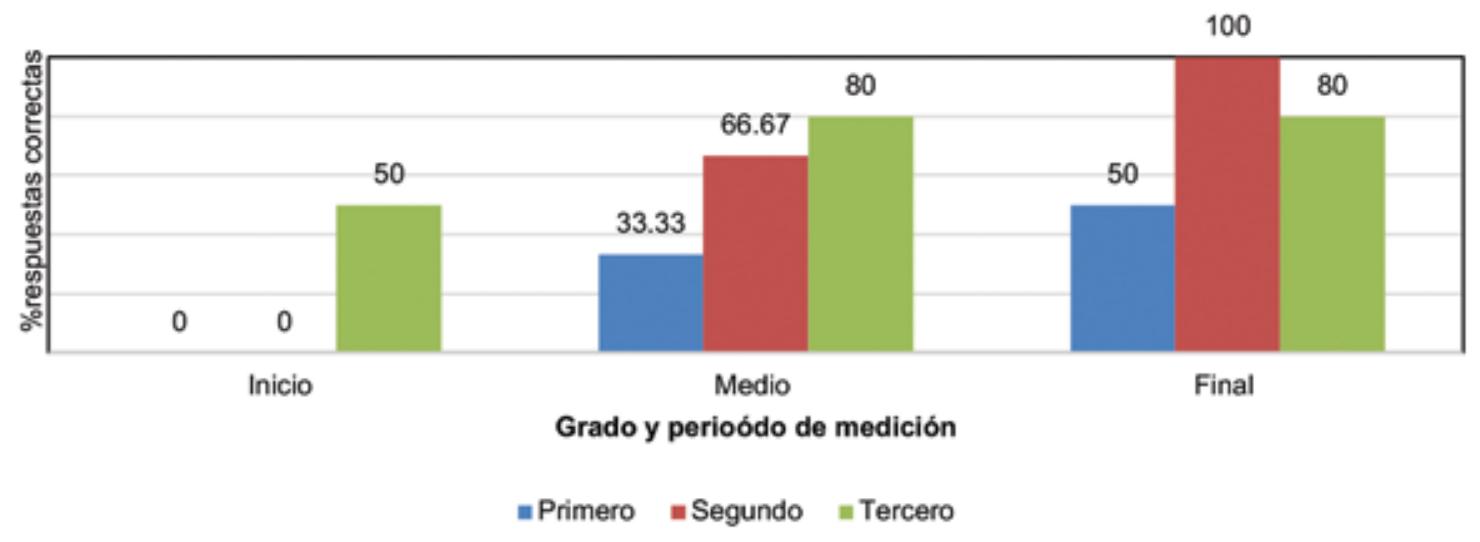

Gráfico 3. Resultados de comprensión lectora por grado y período .

Fuente: Monitoreo y Evaluación del proyecto CARS en la URACCAN. Reporte noviembre 2018.

En relación a las mediciones internacionales, la comprensión lectora en estos niveles educativos corresponde al "75\% de las preguntas respondidas correctamente" (USAID, 2009, pág. 67). De acuerdo con esta medición, se puede afirmar que sólo segundo y tercer grado alcanzan comprender correctamente el contenido de lo que están leyendo. Los niños de primer grado, si bien alcanzan un nivel de comprensión, no llegan al promedio que se requiere según su edad y nivel educativo.

Comparando los promedios de comprensión lectora: se observa un incremento de 23.86 en el 2017 a un 51.11 en el 2018. Este aumento ocurre porque si bien los estudiantes no leían con exactitud las palabras y algunos con un poco de tardanza, en la comprensión ellos respondían correctamente las preguntas.

\section{Lecciones aprendidas}

De los procesos de reflexión con los actores de esta experiencia, resaltan los siguientes aprendizajes y conocimientos: 
1. Mantener una buena comunicación, coordinación y articulación con las autoridades del MINED y la dirección de las escuelas fue clave en el proceso. Esto contribuyó en gran medida, a la colaboración activa con los directores de los diferentes centros escolares para que los planes del equipo técnico de CARS para la implementación de la metodología APA y FAS en los procesos de enseñanza aprendizaje, pudiera combinarse con el plan nacional que manejan los docentes del MINED.

2. Los espacios de reflexión con los padres y madres de familia fueron importantes porque permitió que ellos también asumieran la educación de los niños y la continuidad que se debe dar en el hogar, así también sirvió para identificar problemas y sus posibles soluciones, de esa manera, estuvieron más activos en las escuelas.

3. El hecho de que a los padres se les realizara invitaciones formales de cierta manera aseguró su asistencia en las EPM. Sin embargo, había más asistencia de madres que de padres, porque la mayoría de las veces el hombre va a trabajar al campo, a veces va fuera de la comunidad, mientras las madres siempre están en casa.

4. El acompañamiento a los docentes en la elaboración de materiales didácticos y rincones de aprendizaje ayudó a motivar a los docentes para mantener ambientada las aulas y además la elaboración de materiales móviles.

5. La aplicación de PPL por los docentes fue un nuevo conocimiento que requirió de varios procesos de retroalimentación por parte del equipo técnico, pero al final quedaron fortalecidas las capacidades de los docentes.

6. El uso de metodologías y estrategias educativas, así como el instrumento de evaluación PPL permitió documentar el avance en los resultados de fluidez y comprensión lectora. Los resultados de las PPL sugieren que la fluidez lectora de los niños fue mejorando según el periodo evaluado.

7. Las comisiones de análisis organizadas en las once escuelas fueron fortalecidas por medio de capacitaciones sobre las funciones de cada uno de los miembros para identificar necesidades educativas para que las comisiones quedaran funcionando, aunque el proyecto no continuara.

\section{Agradecimiento}

Esta publicación obtuvo el financiamiento de: El Fondo de Asistencia Internacional de los Estudiantes y Académicos Noruegos (SAIH).

\section{Lista de referencias}

Lopez y Mora. (2016). Guia Metodologica.Lecto-escritura utilizandoel metodo fonico, sintetico (FAS). Managua, Nicaragua: Aula telefonica.

MINED. (2009). Transformación curricular, paradigma y enfoques pedagógicos. Managua, Nicaragua.

Saldarriaga, P. B. (2016). La teoría constructivista de Jean Piaget y su significación para la pedagogía contemporánea. Ciencias Sociales y Políticas, 127-137.

URACCAN . (o1 Febrero al 31 de Diciembre, 2018). Informe del Proyecto CARS. Bilwi, Puerto Cabezas.

URACCAN. (2017). Sendas para recrear y caminar nuestras experiencias con perspectiva intercultural de genero. Nicaragua: Oficina de enlace URACCAN.

USAID. (2009). Manual para la evaluación inicial de la lectura en niños de educación primaria. Islas Canarias. 